Wien klin Mag 2018 ·21:166-171 https://doi.org/10.1007/s00740-018-0240-3 Online publiziert: 16 . August 2018 (c) Der/die Autor(en) 2018

CrossMark

\author{
Karl Kashofer ${ }^{1} \cdot$ Helmut Popper ${ }^{1}$ Luka Brcic $\cdot$ Marija Balic ${ }^{2}$ Ellen Heitzer ${ }^{3}$. \\ Gerald Höfler ${ }^{1}$ \\ ${ }^{1}$ D\&F-Institut für Pathologie, Medizinische Universität Graz, Graz, Österreich \\ ${ }^{2}$ Univ. Klinik für Innere Medizin, Medizinische Universität Graz, Graz, Österreich \\ ${ }^{3}$ D\&F-Institut für Humangenetik, Medizinische Universität Graz, Graz, Österreich
}

\title{
Neue Diagnostik für das Lungenkarzinom
}

\author{
Die Bedeutung von „liquid biopsy“ im \\ Kontext neuer Therapieoptionen
}

Die Diagnostik und Therapie des Lungenkarzinoms ist seit einem Jahrzehnt im Umbruch, immer rascher kommen neue Therapieoptionen hinzu. Viele der neuen Therapien sind sogenannte „targeted therapies" und die behandelnden Onkologen benötigen spezifische Tests zur Vorhersage des Ansprechens des Tumors. Diese Aufgabe kommt verstärkt auf die Pathologie zu und das ständige Entwickeln und Etablieren neuartiger Diagnostik stellt eine der wesentlichen Herausforderungen der modernen Pathologie dar. Neben der klassischen morphologischen Pathologie ist heute die molekulare Diagnostik ein integraler Bestandteil dieser Tätigkeit.

Begonnen hat die neue Ära mit der Entdeckung des EGFR-mutierten Adenokarzinoms der Lunge. Heute sind mehrere Medikamente dafür erhältlich, und neue kommen laufend dazu. Voraussetzung für diese Therapie ist der Nachweis der aktivierenden Mutation im EGFRGen aus der diagnostischen Gewebeprobe.

Sehr bald folgten die Entdeckung der Translokation des ALK-Gens und die Bildung eines Fusionsgens, wie z. B. EML4-ALK. Dieses Fusionsgen führt

Dieser Beitrag stellt eine Zusammenfassung von Vorträgen der Autoren anlässlich der Liquid Biopsy Masterclass dar, die am 23.04.2018in Graz in Kollaboration der Medizinischen Universität Graz mit AstraZeneca Österreich veranstaltet wurde.

Eine Liste mit weiterführender Literatur ist bei den Autoren erhältlich. zur permanenten Aktivierung von ALK und damit zur Proliferation der Adenokarzinomzellen. Auch hier bewirken die Medikamente eine Blockade dieses Gens, und auch hier gibt es mittlerweile eine Reihe höchst wirksamer Substanzen. Die Testung war ursprünglich auf eine Fluoreszenz-in-situ-Hybridisierung(FISH)-Untersuchung beschränkt, mittlerweile wird diese Fusion von ALK mittels Immunhistochemie primär festgestellt und nur im Zweifelsfall folgt eine "polymerase chain reaction" (PCR) auf verschiedene Fusionsgene, die FISH bleibt unklaren Fällen vorbehalten.

Kurz nach Entdeckung der ALK-Fusionen kam die nächste Translokation, nämlich das Fusionsgen mit ROS1. Auch hier erfolgt der Nachweis primär mittels Immunhistochemie, gefolgt von einer PCR-Sequenzierung auf mögliche Fusionsgene. Eine FISH ist auch hier nur problematischen Fällen vorbehalten.

Bei allen diesen genetischen Veränderungen in den Lungenkarzinomen entstehen im Verlauf der Therapie sogenannte Resistenzmechanismen. Eine Reihe verschiedener Mechanismen kennen wir mittlerweile. Häufig sind zum Beispiel Resistenzmutationen im Gen, wodurch die Bindung des Medikaments nicht mehr zustande kommt und dadurch keine Wirkung mehr erzielt werden kann, wie die T790M-Mutation im EGFR. Auch Umgehungsmechanismen sind bekannt, hier übernimmt ein anderes Gen (Tyrosinkinaserezeptor) die Funktion des medikamentös blockier- ten Gens und damit können die Tumorzellen wieder wachsen (z.B. cMET übernimmt die Funktion des EGFR). Es können weiters sekundäre Mutationen auftreten, wodurch auch hier andere Gene die Wachstums-Signalfunktion des blockierten Gens übernehmen (z. B. Inaktivierung von PTEN durch Mutation und dadurch überschießende Wirkung von PI3K-AKT-mTOR). Aber auch hier gibt es viele neue Möglichkeiten: Neue Medikamente können auch solche sekundären Mutationen spezifisch blockieren. Umgehungswege können durch andere Medikamente blockiert werden.

\section{Neues im kleinzelligen neuro- endokrinen Lungenkarzinom}

Lange gab es für das kleinzellige neuroendokrine Lungenkarzinom (SCLC) keine Optionen außer eine Chemo- und/ oder Radiotherapie. Seit Kurzem ist ein neues Medikament auf den Markt gekommen, das für diese Patientengruppe neue Hoffnung bedeutet. Ein Antikörper gegen DLL3 wurde mit einem Toxin gekoppelt. Wenn die Karzinomzelle dieses Molekül an der Oberfläche exprimiert, kann der Antikörper binden, wird in die Zelle aufgenommen, und das Toxin wird abgelöst und kann die Karzinomzelle abtöten. Mittels Immunhistochemie können Pathologen die Expression von DLL3 nachweisen und damit Patienten identifizieren, die von dieser neuen Therapie profitieren können. 


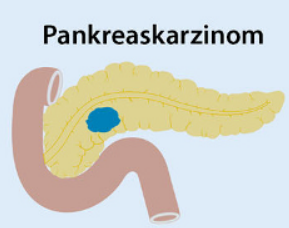

Hochgradiges seröses Ovarialkarzinom

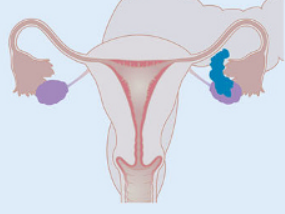

Kolorektales Karzinom

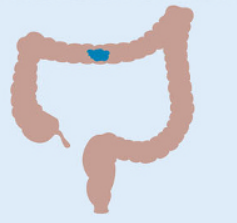

Prostatakarzinom

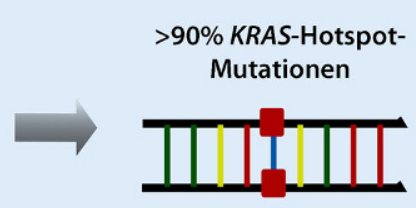

>90\% TP53-Mutationen

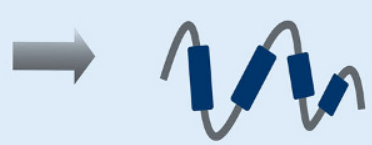

Gene mit wiederkehrender Mutation
Hoch aufgelöstes Profiling einzelner Hotspots

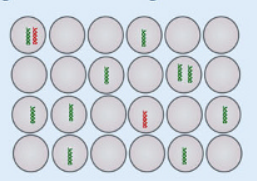

BEAMin

dPCR

LOD bis zu $0,001 \%$

Safe-SeqS, kurze

Barcode-Panels

LOD bis zu $0,01 \%$

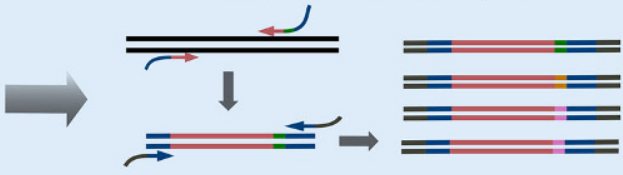

Hoch aufgelöstes Profiling eines Gen-Panels
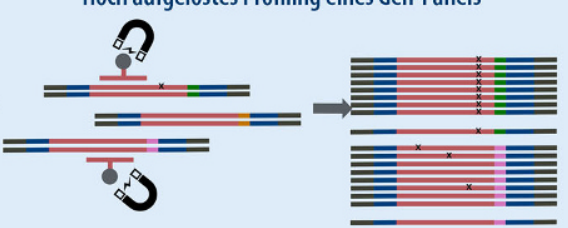

CAPP-Seq

TEC-Seq LOD bis zu $0,0025 \%$

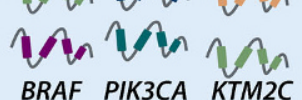

Keine Gene mit wiederkehrender Mutation

Umfassendes nichtgezieltes Profiling
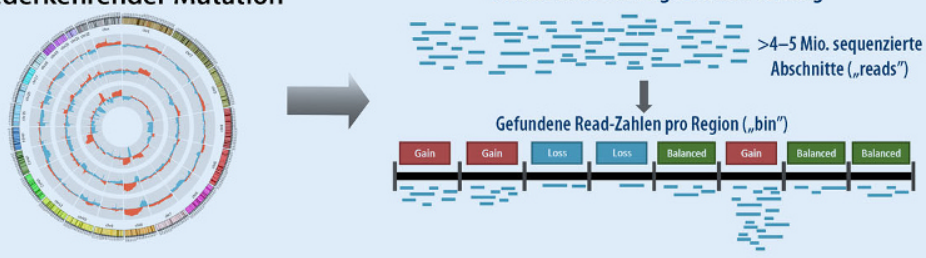

„Whole genome sequencing" mit geringer Abdeckung LOD bis zu 1-5\%

Abb. 1 A Anwendungen der "liquid biopsy“. Unterschiedliche Tumorentitäten stellen verschiedene Anforderungen an die molekulare Analyse. Einzelpunktanalysen können bei Tumoren mit klar definierten Hotspotmutationen verwendet werden. Kleine Genpanels sind besonders in Tumoren mit Mutationen in Tumorsuppressorgenen vorteilhaft und auch größere Genpanels können dank "molecular barcoding“ inzwischen mit hoher Sensitivität analysiert werden. Auch die Bestimmung von Genamplifikationen ist aus der "liquid biopsy" möglich. BEAMing „Beads, emulsion, amplification, magnetics", CAPP-Seq "cancerpersonalized profiling by deep sequencing" (personalisiertes Tumorprofiling durch „deep sequencing"), $d P C R$ digitale PCR, $L O D$ "limit of detection" (Nachweisgrenze), PCR Polymerase-Kettenreaktion, Safe-SeqS "safe sequencing system”, TEC-Seq „targeted error correction sequencing" (Sequenzierung mit gezielter Fehlerkorrektur). (৫ Ellen Heitzer)

\section{Neues im großzelligen neuro- endokrinen Lungenkarzinom}

Für das großzellige neuroendokrine Lungenkarzinom (LCNEC) gab es bisher entweder Operation in frühen Stadien oder Chemotherapie. Seit Jahrzehnten gab es eine Diskussion, welche Art von Chemotherapie besser sei: das Schema für das SCLC oder das für Adenokarzinome. Dies scheint nun gelöst zu sein. LCNEC, die einen Verlust des RB1-Gens aufweisen, profitieren von einer Chemotherapie mittels SCLC-Schema, während LCNEC mit einem Verlust von PTEN oder einer aktivierenden Mutation des PI3KCA-Gens von einer klassischen Cisplatin basierten Chemotherapie pro- fitieren. LCNEC exprimieren ebenfalls DLL3 und könnten möglicherweise auch auf diese Therapie ansprechen. Die Testung dieser Gene erfolgt wieder mittels Immunhistochemie. 1

Ursprüngliche Hoffnungen das Plattenepithelkarzinom mittels einer Blockade von FGFR1 therapieren zu können, haben sich leider nicht bewahrheitet. Diese Karzinome besitzen zumeist weitere mutierte Wachstumsrezeptoren, die bei Blockade von FGFR1 sofort die Funktion übernehmen.

\section{Immuntherapie}

Schon vor Jahrzehnten gab es Versuche, das menschliche Immunsystem in den Kampf gegen Karzinome einzubeziehen. Aber erst seit wenigen Jahren ist hier ein Durchbruch in der Forschung gelungen. Mit der Entdeckung von sogenannten Checkpoint-Inhibitoren, wie CTLA4 und PD1-PDL1 hat eine neue Ära in der Immuntherapie eingesetzt. Viele Lungenkarzinome exprimieren an ihrer Oberfläche PDL1 (Ligand des Todrezeptors PD1) und verhindern damit, dass zytotoxische Lymphozyten die $\mathrm{Tu}$ morzelle angreifen. Durch eine Blockade des PDL1 oder auch PD1 wird aber nun die Kommunikation zwischen Tumorzelle und Lymphozyten blockiert, und die Lymphozyten greifen nun wieder die Karzinomzellen an und vernichten sie. Der 
Nachweis von PDL1-Expression erfolgt mittels Immunhistochemie.

Der Einsatz vieler neuer Medikamente kann aber nur erfolgen, wenn die aktivierende Mutation oder die Resistenzmutation im Tumor des Patienten nachgewiesen werden kann. Da bei Patienten in fortgeschrittenem Stadium eine Gewebeentnahme durch Biopsie häufig nicht mehr möglich ist, besteht hier dringender Bedarf nach alternativem Probenmaterial, eine Lücke, die die sogenannte „liquid biopsy“ unter Umständen füllen kann. Unter „liquid biopsy“ versteht man die Analyse von Tumorbestandteilen aus eine Blutprobe des Patienten. Die Blutprobe enthält zwei Arten von Tumormaterial, die zirkulierenden Tumorzellen und die zellfreie zirkulierende Tumor-DNA.

\section{Zirkulierende Tumorzellen}

Das Bestreben, die metastasierenden Tumorzellen anzureichern, beruht auf den Studien aus den frühen 1980er-Jahren, mit den ersten Hinweisen, dass epitheliale Zellen in Knochenmarksaspiraten und Lymphknoten der Patientinnen mit Brustkrebs gefunden werden können, und vor allem, dass die Präsenz dieser Zellen im Knochenmark mit einer schlechteren Prognose der Patientinnen einhergehen. In der Meta-Analyse von Braun et al. (2005) und auch in der ACOSOG Z0010 Studie konnte die prognostische Signifikanz dieser Zellen gezeigt werden $[1,2]$. Der initiale Nachweis dieser disseminierten Tumorzellen hat das Bestreben erweckt die zirkulierenden Tumorzellen für eine diagnostische Aussage zu analysieren. Es führte zur Entwicklung unterschiedlicher Anreicherungs- und Detektionsmethoden, ein Beispiel ist die größenbasierte Selektion mit dem anschließenden Nachweis der epithelialen Zytokeratin-Marker der Tumorzelle. Mit dieser Methode können zirkulierende Tumorzellen im Hintergrund der Blutzellen, vor allem der weißen Blutkörperchen, erkannt und aufgereinigt werden. Die zirkulierenden Tumorzellen treten in einer Häufigkeit von 1 Zelle in $1 \mathrm{ml}$ Blut [3] auf. Die CellSearch Methode, eine auf Anreicherung von EpCAM-positiven Zellen ausgerichtete elektromagnetische An-

Wien klin Mag 2018 $21: 166-171 \quad$ https://doi.org/10.1007/s00740-018-0240-3

(c) Der/die Autor(en) 2018

\author{
K. Kashofer · H. Popper · L. Brcic · M. Balic · E. Heitzer · G. Höfler
}

Neue Diagnostik für das Lungenkarzinom. Die Bedeutung von "liquid biopsy“ im Kontext neuer Therapieoptionen

\section{Zusammenfassung}

Die molekulare Diagnostik des Lungenkarzinoms hat in den letzten Jahren eine rasante Entwicklung erfahren. Neue molekulare Targets, Resistenzmutationen für bestehende Therapien und nicht zuletzt die Immuntherapie rücken die molekulare Diagnostik immer mehr in den Mittelpunkt. Die Entdeckung der zirkulierenden Tumorzellen und der freien zirkulierenden Tumor-Desoxyribonukleinsäure (ctDNA) hat besonders für das Lungenkarzinom aufgrund der eingeschränkten Verfügbarkeit von Gewebeproben hohe Bedeutung. Inzwischen ist der Nachweis von Tumormutationen aus dem Blut gut etabliert und kann zur Beurteilung der Tumorlast und auch für die Detektion von Resistenzmutationen verwendet werden. Trotzdem zeigt sich, dass die Analyse von ctDNA technisch aufwendig und komplex ist und dass nicht in allen Tumorstadien und Tumorentitäten ctDNA im Patienten nachweisbar ist. Die neuesten Entwicklungen in der Diagnostik des Lungenkarzinoms und die Bedeutung der "liquid biopsy" in diesem Kontext sind Thema dieses Artikels.

\section{Schlüsselwörter}

Lungenkrebs - Next-Generation Sequencing - Zirkulierende Tumor-DNA (ctDNA) · Zirkulierende zellfreie DNA (ccfDNA) . Molekularpathologie

\title{
New Diagnostics in Lung Cancer. The Significance of "Liquid Biopsy" in the Context of Developments of New Treatment Options
}

\section{Abstract}

During the past few years, molecular methods in the diagnosis of lung cancer have undergone rapid development. New molecular targets, resistance mutations in existing therapies, and, not least, immunotherapy, have placed molecular diagnosis center stage. Particularly in the case of lung cancer, because of the limited access to tissue samples, the discovery of circulating tumor cells and circulating tumor DNA (ctDNA) is of great importance. Meanwhile, evidence for tumor mutations from the blood is wellestablished and can be utilized for evaluation of the tumor burden and for the detection of resistance mutations. Nevertheless, analysis of ctDNA is technically elaborate and complex and it is not detectable in patients at all tumor stages and in all tumor entities. The most recent developments in the diagnosis of lung cancer and the significance of "liquid biopsy" in this context are discussed in this article.

\section{Keywords}

Lung cancer · Next-Generation Sequencing Circulating tumor DNA - Circulating cell-free DNA · Molecular pathology reicherung, wurde sogar von der FDA (Food and Drug Administration Federal Agency) zur Detektion von zirkulierenden Tumorzellen genehmigt. Mit dieser Methode wurde vor allem in Brustkrebs gezeigt, dass zirkulierende Tumorzellen prognostisch ungünstig sind und sich als Methode eignen, den Therapieerfolg zu monitieren. Insbesondere Patientinnen, die sich mit erhöhter Anzahl an Tumorzellen nach begonnener Therapie beim zweiten Zyklus präsentierten, hatten eine schlechte Prognose [4]. Obwohl diese Studien bisher keine klinische Relevanz im Sinne von Patientenmanagement und Therapiewechsel belegen konnten, wurde das Interesse an der „liquid biopsy“ wesentlich von den durchgeführten Studien bewegt. Auch in den frühen Tumorstadien konnte die prognostische Signifikanz des Nachweises von zirkulierenden Tumorzellen gezeigt werden. Bei operablen Lungenkarzinompatienten ist der Nachweis von zirkulierenden Tumorzellen unabhängig von Lymphknotenmetastasen prognostisch ungünstig und konnte eine höhere Detektionsrate als die Analyse der freien zirkulierenden DNA bei Patienten mit Lungenadenokarzinomen erzielen [5]. 


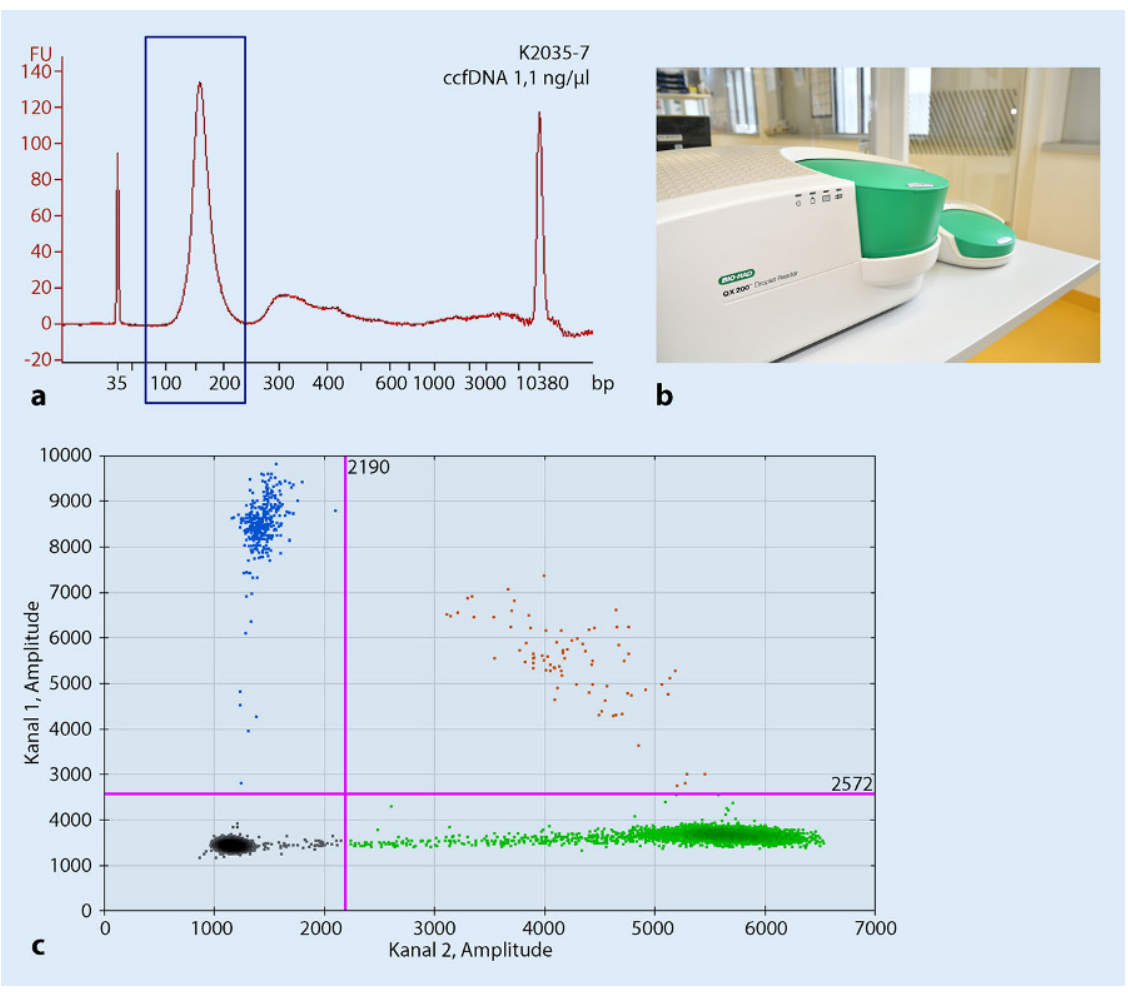

Abb. 2 \ Mutationsnachweis mit ddPCR. a Extrahierte ctDNA ist im Elektropherogramm als Signal bei 160 bp sichtbar. b Digitale Droplet PCR (im Bild QX200, BioRad) partitioniert eine mutationsspezifische PCR-Reaktion in 22.000 Einzelreaktionen. c Die Fluoreszenz der einzelnen Droplets gibt Aufschluss über den EGFR T790M-Mutationsstatus. Die Droplets mit positiver Mutationsdetektion befinden sich im linken oberen Quadranten (blau), doppelt positive im rechten oberen Quadranten (orange), Droplets mit negativem Mutationsstatus im unteren rechten Quadranten (grün). ccfDNA "Circulating cell-free DNA" (zirkulierende freie DNA). (@ Karl Kashofer)

Dabei verwendeten wir den Mikrofilter zur größenbasierten Selektion, der in einer früheren Studie als dem CellSearch System überlegen gezeigt wurde. Der in dieser Studie verwendete Mikrofilter bietet einige Möglichkeiten zur Analyse der zirkulierenden Tumorzellen nach Anreicherung und ist dem CellSearch System in einigen Aspekten überlegen [6]. Die weiteren möglichen Anwendungen der Analyse von zirkulierenden Tumorzellen werden aktuell untersucht und besonders interessant sind dabei natürlich Vergleiche mit den Protokollen zur Analyse der freien zirkulierenden TumorDNA mittels optimierter NGS (NextGeneration-Sequencing)-Ansätze.

\section{Zellfreie zirkulierende Tumor-DNA}

Die „liquid biopsy“, auf Deutsch auch „Flüssigbiopsie“ genannt, gewinnt in der Onkologie zunehmend an Bedeutung.
ccfDNA enthält und diese freie DNA stark fragmentiert ist, stellt die Analytik vor große Herausforderungen. Zudem kann der Anteil der tumorspezifischen DNA (ctDNA, circulating tumor DNA) stark variieren, bei einigen Tumorpatienten findet man 50-90\% Tumor-DNA im Plasma, bei wiederum anderen weniger als $1 \%$, wobei Patienten in früheren Stadien generell nur wenig $(<1 \%)$ vom Tumor stammende DNA-Fragmente in der Zirkulation aufweisen.

Dementsprechend haben sich eine ganze Reihe von Analysemethoden entwickelt, mit denen man einerseits eine einzelne Zielregion mit sehr hoher Auflösung analysieren kann, aber auch umfassende, genomweite Analysen durchführen kann (•Abb. 1). Die Auswahl der Analysemethode richtet sich nach Tumorstadium, Biologie und klinischer Anwendung. Anhand tumorspezifischer Mutationsanalysen kann man beispielsweise Rezidive frühzeitig, noch bevor diese klinische evident werden, detektieren. Ebenso dient der Nachweis von ctDNA nach kurativer Operation oder vor Initiierung einer neuen Therapie als negativer prognostischer Marker und es wurde gezeigt, dass der Level an ctDNA mit dem Therapieansprechen korreliert. Das Aufkommen von neuen Therapiezielen bzw. Resistenzmechanismen kann ebenfalls mittels ctDNA nachgewiesen werden und so das Therapiemanagement von Tumorpatienten erleichtern.

Im konkreten Beispiel des Lungenkarzinoms ist es vor allem die T790M-Resistenzmutation des EGFR-Gens, die diese Art der Analytik in Rollen brachte. Im November 2015 wurde von der FDA die Zulassung von Osimertinib (Tagrisso ${ }^{\circledR}$, AstraZeneca PLC, London, UK) erteilt. Dieses Medikament ist ein Vertreter der sogenannten „targeted therapies“; es wurde entwickelt, um das Wachstum von Tumorzellen einzuschränken, die unter Tyrosinkinasetherapie bei aktiviertem EGFR die spezifische Resistenzmutation Thr790Met im Exon 20 des EGFR entwickelt haben. In diesen Tumorzellen ist die Bindung der klassischen Tyrosinkinaseinhibitoren wegen der Resistenzmutation nicht mehr möglich. Osimertinib ist in seiner dreidimensionalen Struktur optimiert, um genau an 


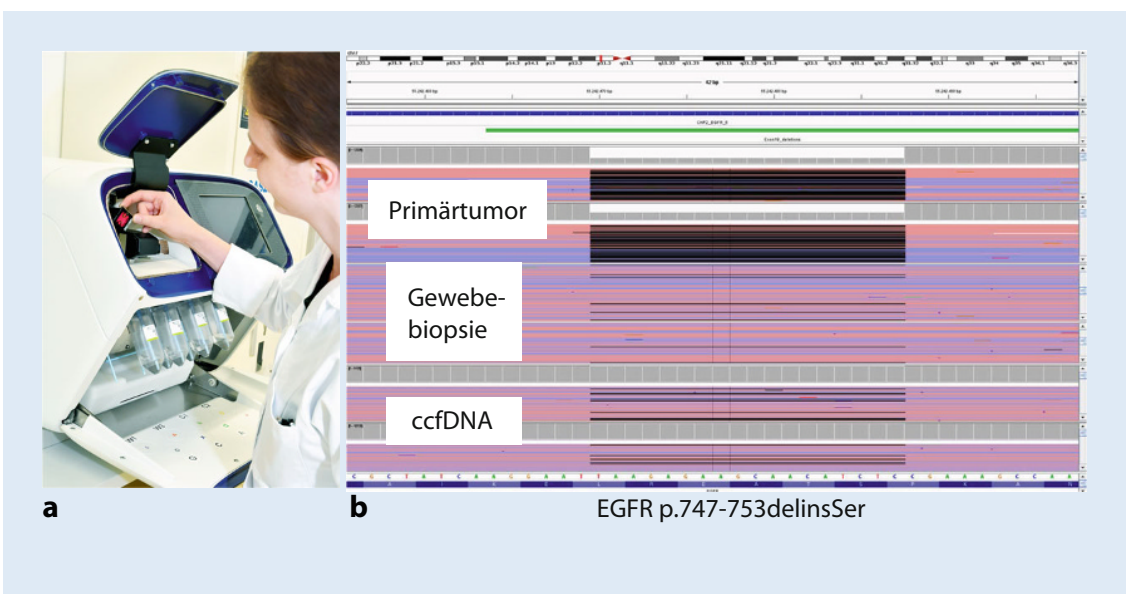

Abb. 3 a ccfDNA-Analyse mit Next-Generation-Sequencing (NGS). a Die lon Torrent NGS-Plattform von Thermo Fisher basiert auf der Messung von Veränderungen des $\mathrm{pH}$-Wertes durch den Einbau von Nukleotiden in den DNA-Strang. Sie kann bis zu 150 Mio. Moleküle pro Durchlauf sequenzieren und bietet über die Oncomine ccfDNA Assays auch standardisierte Tests für „liquid biopsy“. b Die EGFR Exon 19 Deletion kann mittels NGS sowohl im Primärtumor als auch in der Gewebebiopsie und im Plasma nachgewiesen werden. (๑ Karl Kashofer)

das durch diese spezifische Resistenzmutation veränderte Protein anzudocken und dadurch die Kinaseaktivität des EGF-Rezeptors zu blockieren. Um die Therapie von Patienten von klassischen Tyrosinkinaseinhibitoren auf Osimertinib umzustellen und auch um die Zulassungsbedingungen $\mathrm{zu}$ erfüllen, ist der Nachweis der Resistenzmutation T790M notwendig. Da eine Gewebebiopsie bei Patienten im fortgeschrittenen Stadium oft nicht mehr möglich ist, wurde hier schon früh die Analyse der zirkulierenden Tumor-DNA etabliert. In den 2018 neu herausgegebenen internationalen Richtlinien für die molekulare Analyse von Lungenkarzinomen wird bereits angeführt, dass die ccfDNA für die Bestimmung des EGFR-Mutationsstatus verwendet werden kann, wenn bereits eine histologische Diagnose des Tumors besteht und kein weiteres $\mathrm{Tu}$ mormaterial verfügbar ist. Bei Patienten, die unter Tyrosinkinaseinhibitortherapie Tumorprogression zeigen, ist die Analyse der ccfDNA ebenfalls schon zulässig; bei negativem Ergebnis sollte die Analyse aber aus Tumorgewebe wiederholt werden.

\section{ctDNA-Analysemethoden}

Prinzipiell muss für die Analyse der ctDNA initial eine optimale Asservierung der Patientenblutprobe gewährleis- tet werden. Die Hauptkontaminationsquelle von ctDNA ist die genomische DNA von weißen Blutkörperchen, die durch falsche Asservierung bersten und ihre genomische DNA in das Patientenplasma abgeben. Diesem Zelltod kann durch schonende Fixierung der zellulären Bestandteile der Blutprobe vorgebeugt werden. $\mathrm{Zu}$ diesem Zweck sind am Markt mehrere Abnahmesysteme mit speziellen Stabilisierungslösungen etabliert. Derzeit sind vor allem die Systeme von Streck (Cell-Free DNA BCT, Omaha, USA), Qiagen (PAXgene Blood ccfDNA Tube, Hilden, Deutschland) und Roche (Cell-Free DNA Collection Tube, Basel, Schweiz) in Verwendung. Mit all diesen Abnahmeröhrchen kann eine ausreichende Stabilisierung der zirkulierenden DNA für einige Tage bei Raumtemperatur erreicht und damit eine praxistaugliche Logistik aufgebaut werden. Die Extraktion der ctDNA aus der Blutprobe muss vor allem eine möglichst hohe Effizienz aufweisen, um nicht schon in diesem Schritt Tumor-DNAMoleküle und damit analytische Sensitivität zu verlieren. In unseren Händen haben sich die Extraktionssysteme von Qiagen (QIAamp Circulating Nucleic Acid Kit) und Promega (Maxwell RSC ccfDNA Plasma Kit, Madison, US) bewährt. Die Extraktion der ctDNA liefert zwischen 5 und 300 ng an fragmentierter DNA mit einer Länge von ungefähr 160
Nukleotiden, die aus der Apoptose und anderen Zelluntergängen von epithelialen Zellen des Patienten und auch der Tumorzellen hervorgeht (• Abb. 2a).

Die Analyse der zirkulierenden DNA erfordert hohe Sensitivität, da oft nur ein geringer Prozentsatz der extrahierten DNA vom Tumor stammt. In der Praxis sind derzeit drei Analysemethoden im Einsatz. Die quantitative PCR mit mutationsspezifischen Primern (z. B. Roche cobas Tests) ist in der Primärdiagnose gut etabliert und kann auch für die ctDNA verwendet werden. Probleme ergeben sich durch die vergleichsweise geringe Sensitivität der Methode die mit $\sim 1 \%$ mutierten Allelen angegeben wird. Nur wenige Lungenkarzinome zeigen mutierte ctDNA in so hoher Konzentration, was die Gefahr von falsch-negativen Befunden mit sich bringt. Eine weitere Methode zur Analyse von ctDNA stellt die digitale PCR dar. In diesem Verfahren wird eine mutationsspezifische PCR in Mikrotröpfchen aufgeteilt und in jedem Mikrotröpfchen eine EndpunktPCR durchgeführt. Dieses sehr starke Signal wird dann in den Einzelreaktionen vermessen und die Anzahl der positiven Reaktionen in Relation zu den negativen Reaktionen gesetzt. Derzeit gibt es zwei Systeme für digitale PCR, das QuantStudio 3D Digital PCR System von Thermo Fisher (Waltham, USA) und das QX200 System von BioRad (Hercules, USA; - Abb. 2b, c). Beide Systeme sind in der Lage bei einem gut optimierten Assay und ausreichendem Probenmaterial ein mutiertes Allel im Hintergrund von 10.000 nicht mutierten Allelen nachzuweisen (0,01 Sensitivität). Die digitale PCR ist damit wesentlich sensitiver als die klassische quantitative PCR und kann trotzdem in sehr kurzer Zeit, typischerweise einem Arbeitstag, durchgeführt werden.

Trotz dieser Vorteile der digitalen PCR ist diese Methode noch immer eine Einzelpunktanalyse, das heißt, dass immer nur ein einzelner Mutationshotspot analysiert werden kann. Die Methode ist deshalb für die Verlaufskontrolle, um zum Beispiel das Therapieansprechen zu beobachten, gut geeignet, kann aber eine breite Analytik für das Auftreten diverser Resistenzmutationen nicht ersetzen. 


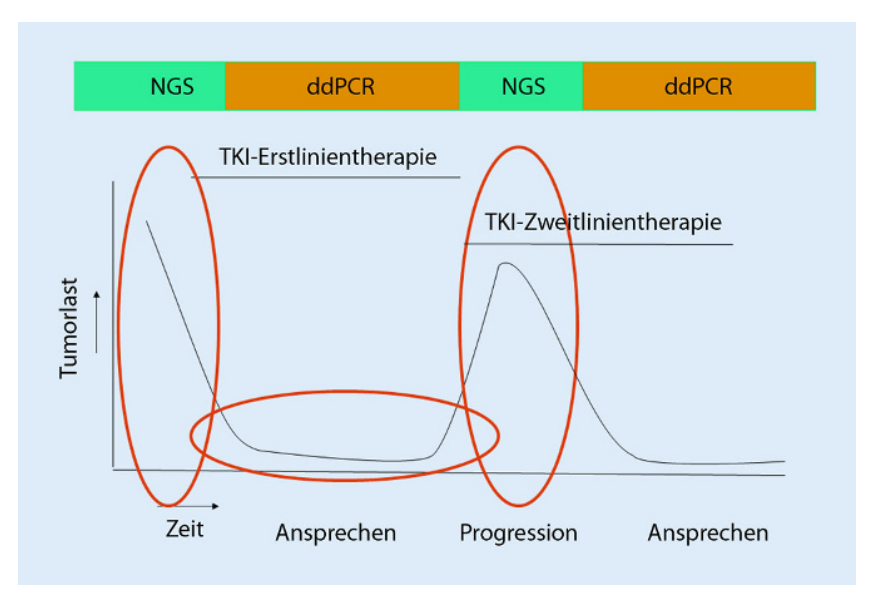

Abb. 4 ॥ Analyseschema für rezidivierende Tumorerkrankungen. Tumorlast und Resistenzmutation benötigen unterschiedliche Analysemethoden. In der Progression ist eine breite NGS-Analytik, die sämtliche relevanten Resistenzmutationen beinhaltet, notwendig, aber auch technisch aufwendig und kostenintensiv. In den dazwischenliegenden Intervallen kann die Tumorlast mittels ddPCR für eine Hauptmutation des Tumors bestimmt werden. $d d P C R$ Digitale Droplet-Polymerase-Kettenreaktion, $N G S$ "next generation sequencing“. (๑ Karl Kashofer)

In dieser Fragestellung ist das NGS die aktuell beste Methode( $\bullet$ Abb. 3). Beim NGS werden parallel sehr viele DNA-Moleküle analysiert und es kann damit ein breites Mutationsprofil erstellt werden. Die notwendige hohe Sensitivität kann durch sogenanntes „molecular barcoding“, also die molekulare Markierung von individuellen Einzelmolekülen erreicht werden. Diese Art von Assays ist inzwischen für beide großen NGS-Plattformen (Illumina, San Diego, USA und Thermo Fisher) erhältlich und in Graz bereits seit mehr als einem Jahr Bestandteil der Routinediagnostik. Mit dem Oncomine Lung cfDNA Assay von Thermo Fisher können über 150 Mutationshotspots mit hoher Sensitivität $(0,1 \%$ mutierte Allelfrequenz) ausgelesen werden und die Therapie des Lungenkarzinoms damit auf die dynamischen Veränderungen im Zeitablauf angepasst werden (• Abb.4).

Die Anwendung der „liquid biopsy“ist ein Forschungsschwerpunkt der Medizinischen Universität Graz und derzeit laufen Studie in vielen Tumorentitäten, um die Anwendbarkeit und vor allem auch die klinische Bedeutung dieser Analytik zu belegen. Diese Forschung ist dringend notwendig, denn trotz aller vielversprechenden Ansätze lassen tatsächliche breite klinische Anwendungen bisher auf sich warten. Mit der Ausnahme der EGFRTestung aus Plasma bei Lungenkrebspatienten hat es keine der bisherigen An- wendungen in die Klinikgeschafft. Daher ist es unabdingbar, die Sensitivität und Genauigkeit bzw. den prädiktiven und prognostischen Wert der ctDNA in großen prospektiven Studien zu evaluieren und gemeinsame Standards für die Analyse der ctDNA zu etablieren.

\section{Korrespondenzadresse}

Mag. Dr. K. Kashofer

D\&F-Institut für Pathologie, Medizinische Universität Graz

Neue Stiftingtalstraße 6, 8010 Graz, Österreich karl.kashofer@medunigraz.at

Funding. Open access funding provided by Medical University of Graz.

\section{Einhaltung ethischer Richtlinien}

Interessenkonflikt. K. Kashofer, H. Popper, L. Brcic, M. Balic, E. Heitzer und G. Höfler geben an, dass kein Interessenkonflikt besteht.

Dieser Beitrag beinhaltet keine von den Autoren durchgeführten Studien an Menschen oder Tieren.

Open Access. Dieser Artikel wird unter der Creative Commons Namensnennung 4.0 International Lizenz (http://creativecommons.org/licenses/by/4.0/deed. de) veröffentlicht, welche die Nutzung, Vervielfältigung, Bearbeitung, Verbreitung und Wiedergabe in jeglichem Medium und Format erlaubt, sofern Sie den/die ursprünglichen Autor(en) und die Quelle ordnungsgemäßnennen, einen Link zur Creative Commons Lizenz beifügen und angeben, ob Änderungen vorgenommen wurden.

\section{Literatur}

1. Braun S et al (2005) A pooled analysis of bone marrow micrometastasis in breast cancer. $\mathrm{N}$ Eng J Med 353:793-802. https://doi.org/10.1056/ NEJMoa050434

2. Guiliano AE et al (2011) Association of occult metastases in sentinel lymph nodes and bone marrow with survival among women with earlystage invasive breast cancer. J Am Med Assoc 306(4):385-393

3. Balic M et al (2013) Circulating tumor cells: from bench to bedside. Annu Rev Med. https://doi.org/ 10.1146/annurev-med-050311-163404

4. Cristofanilli M et al (2005) Circulating tumor cells, disease progression, and survival in metastatic breast cancer. N Engl J Med 351(8):781-791

5. Dandachi N et al (2017) Frequency and clinical impact of preoperative circulating tumor cells in resectable non-metastatic lung adenocarcinomas. Lung cancer. https://doi.org/10.1016/j.lungcan. 2017.10.003

6. Lin HKet al (2010) Portable filter-based microdevice for detection and characterization of circulating tumor cells. Clin Cancer Res 16(20):5011-5018

7. Mandel P, Metais $P$ (1948) Les acides nucléiques du plasma sanguin chez l'homme. C R Seances Soc Biol Fil 142(3-4):241-243 